\title{
The improvement of liposome-mediated transfection of pEGFP DNA into human prostate cancer cells by combining low-frequency and low-energy ultrasound with microbubbles
}

\author{
WEN-KUN BAI*, ZUO-HUI WU**, E SHEN, JI-ZHEN ZHANG and BING HU \\ Department of Ultrasound in Medicine, Shanghai Jiao Tong University Affiliated 6th People's Hospital, \\ Shanghai Institute of Ultrasound in Medicine, Shanghai 200233, P.R. China
}

Received August 25, 2011; Accepted September 30, 2011

DOI: 10.3892/or.2011.1510

\begin{abstract}
The aim of this study was to explore the use of a contrast agent to study the effects of exposure to ultrasound, in combination with microbubbles, on liposome-mediated transfection of genes into human prostate cancer cells. A contrast agent was used to study the effects of ultrasound exposure in combination with microbubbles on liposomes, which transfect genes into human prostate cancer cells. The human prostate cancer cell line PC-3 in suspension was exposed to ultrasound with a $20 \%$ duty cycle (i.e., $2 \mathrm{sec}$ 'on' time and $8 \mathrm{sec}$ 'off' time) lasting $5 \mathrm{~min}$, with and without ultrasound contrast agent (SonoVue ${ }^{\mathrm{TM}}$ ) using a digital sonifier at a frequency of $21 \mathrm{kHz}$ and an intensity of $4.6 \mathrm{~mW} / \mathrm{cm}^{2}$. Immediately after exposure to ultrasound, cell viability and membrane damage were measured. After exposure to ultrasound, the cell suspensions were put into 12 -well plates and cultured for $24 \mathrm{~h}$. Fluorescence microscopy and flow cytometry were used to detect pEGFP transfection efficiency. Exposure to ultrasound alone and ultrasound combined with microbubbles resulted in minimal cell death and induced negligible cell membrane damage. Ultrasound combined with microbubbles had a greater effect on cell membrane damage in all groups: the average cell membrane damage was $41.87 \%$, and it was approximately 42-fold greater than in the control group. The average transfection efficiency of PC-3 cells was $20.30 \%$ for the liposome $\left(\right.$ Lipofectamine $\left.^{\mathrm{TM}}\right)+\mathrm{pEGFP}+$ ultrasound+ultrasound contrast agent (SonoVue) group; this was the highest rate of all groups
\end{abstract}

Correspondence to: Dr Bing Hu, Department of Ultrasound in Medicine, Shanghai Jiao Tong University Affiliated 6th People's Hospital, Shanghai Institute of Ultrasound in Medicine, Yishan Road 600, Shanghai 200233, P.R. China

E-mail: bing-hu021@hotmail.com

*Shared senior authorship

Key words: low-frequency ultrasound, low-energy ultrasound, liposome, microbubble, transfection, gene therapy measured and was approximately 81 -fold greater than that of the control group. The use of low-frequency and low-energy ultrasound, in combination with microbubbles, could be a potent physical method for increasing liposome gene delivery efficiency. This technique is a promising non-viral approach that can be used in prostate cancer gene therapy.

\section{Introduction}

Prostate cancer was ranked first in incidence rate of cancers among men in the US in 2010, and its mortality was ranked second, behind lung cancer (1). The main methods of therapy used to treat prostate cancer are surgery, radiation and hormone therapy $(2,3)$. These methods have certain curative effects as therapies for cancer, but they still have some limitations, such as injury to surrounding tissues, drug resistance and recurrence (3-5).

Gene therapy is a promising method for the treatment of human diseases. Among many other anticancer treatments, gene therapy has gained attention in clinical trials for its low incidence of side effects, compared to chemotherapy and radiotherapy (6). Several methods have been developed for the delivery of DNA into cells. These methods include chemically facilitated, vector-mediated, mechanical (7) and electric pulse methods (8). In current clinical protocols for gene therapy, virus-derived vectors and non-virus-derived vectors have been used in most trials (9). Although viral vectors have high transfection efficiencies over a wide range of cell targets, they have major limitations, such as immune responses to viruses and insertional mutagenesis, when used as vectors in clinical trials (10). These unwanted side effects have drawn attention to non-viral methods of gene transfer. Non-viral vectors, such as liposome-mediated gene transfer, are attractive alternatives to viral vectors due to their safety, versatility and ease of preparation and scale-up. However, non-viral vectors generally suffer from relatively low transfection efficiencies $(11,12)$. The combination of ultrasound with microbubbles is also a nonviral vector to mediate gene transfection and can possibly be applied for clinical use because it is considered easier and safer than other methods; also, it could strengthen the effect of the 
non-viral methods of gene transfer. Collapsing microbubbles and the cavitation bubbles created by this collapse generate impulsive pressures, such as liquid jets and shock waves, that cause transient membrane permeability and allow exogenous molecules to enter cells. These pressures also affect neighboring cells. The shock-wave propagation distance from the center of a cavitation bubble that has the potential to damage the cell membrane is considerably greater than the maximum radius of the cavitation bubble (13).

In this study, we evaluated the improvement of liposomemediated transfection of green fluorescent protein (pEGFP) DNA into human prostate cancer cells by low-frequency and low-energy ultrasound combined with microbubbles. The aims of the study were to elucidate the mechanism by which ultrasound combined with microbubbles improves liposome-mediated gene transfer that might be used to treat prostate cancer.

\section{Materials and methods}

This study obtained permission from the ethics committee of the Shanghai Jiao Tong University Affiliated 6th People's Hospital and the Shanghai Institute of Ultrasound in Medicine.

Cell culture. In order to study solid prostate cancer tumor-cell transfection at the cellular level, we used a human prostate cancer cell line, PC-3, which was obtained from the Cell Bank of the Chinese Academy of Sciences (Shanghai, China). The cells were grown in DMEM (Gibco, Grand Island, NY, USA) supplemented with $10 \%$ heat-inactivated fetal bovine serum (Invitrogen, Carlsbad, CA, USA) at $37^{\circ} \mathrm{C}$ in humidified air containing $5 \% \mathrm{CO}_{2}$. In the experiment, $\mathrm{PC}-3$ cells were resuspended and counted for a density of $1 \times 10^{5}$ cells $/ \mathrm{ml}$, and then they were put into $1.5-\mathrm{ml}$ polystyrene sample test tubes, which showed no significant effect on the acoustic permeation ratio when exposed to ultrasound. Each tube contained a 1-ml suspension of PC-3 cells. The diameter of the tubes was $13 \mathrm{~mm}$, and the tube bottoms were planar, which allowed them to be placed more closely to the ultrasound probe.

Ultrasound apparatus and microbubbles. Gene transfection was performed by FS-450 ultrasonic processing (Shanghai Institute of Ultrasound in Medicine, China) using a SonoVue $^{\mathrm{TM}}$ microbubble echo-contrast agent (Bracco SpA, Milan, Italy). The FS-450 ultrasonic processor was equipped with a built-in digital timer, intensity regulator and duty factor controller; the duty cycle could range from 10 to $90 \%$, and the probe frequency was fixed at $21 \mathrm{kHz}$. The peak acoustic amplitude in degassed water was measured using a calibrated poly-(vinylidene difluoride-trifluoroethylene) needle-type hydrophone, $0.5 \mathrm{~mm}$ in diameter (Toray Techno Co., Ltd., Japan), connected to a PC/AT-compatible computer and a digitizing oscilloscope (TDS3034, Tektronix Japan, Ltd., Japan). The spatial-average temporal average intensity (ISATA) and peak acoustic pressure corresponding to the reading output were $4.6 \mathrm{~mW} / \mathrm{cm}^{2}$ and $0.007 \mathrm{MPa}$, respectively. In all studies, ultrasound was generated by a $21-\mathrm{kHz}$ ultrasound probe, and the duty cycle was $20 \%$ (i.e., $2 \mathrm{sec}$ 'on' time and $8 \mathrm{sec}$ 'off' time). The exposure time was $5 \mathrm{~min}$. The shape of the probe was cylindrical, and the diameter of the ultrasound probe was $13 \mathrm{~mm}$, which was the same as the diameter of the test tubes.

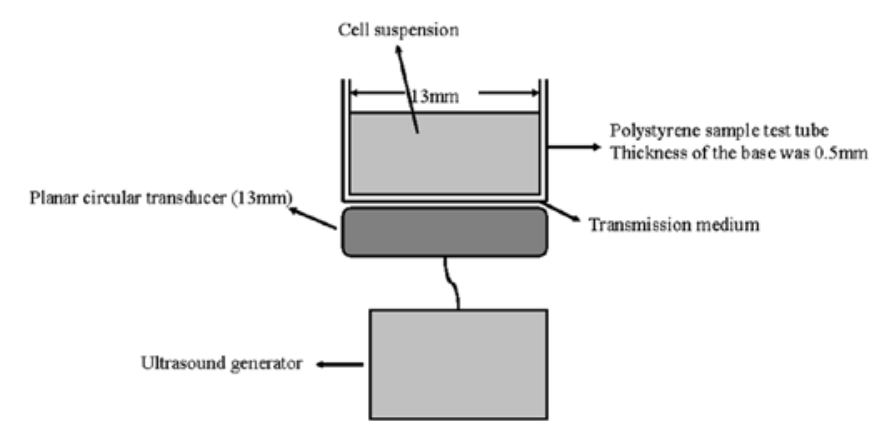

Figure 1. Experiment setup for ultrasound exposure.

In all experiments, the clamp was attached to a metal stand to keep the transducer facing directly upward. The tube was placed on the center of a transducer intermediated with gel (Fig. 1). This setup allowed for standing wave formation due to the reflection of ultrasound radiation at the water-air interface (14). We used a near acoustic field produced by the liquid-air interface and did not reduce standing waves because of the efficient occurrence of cavitation (15).

The SonoVue agent used was a lipid-shelled ultrasound contrast agent composed of microbubbles filled with sulfur hexafluoride gas. The microbubbles were 2.5-6.0 $\mu \mathrm{m}$ in diameter. Upon use, the SonoVue was reconstituted in $5 \mathrm{ml}$ phosphate-buffered saline (PBS) that contained $2-5 \times 10^{8}$ microbubbles $/ \mathrm{ml}$.

Preparation of plasmid DNA. The pEGFP plasmid DNA (donated by Dr Cui-Xia Yang, Center Laboratory of Shanghai Jiao Tong University Affiliated 6th People's Hospital, China) was prepared with a special reagent (E.ZN.A Plasmid Miniprep kit II, Omega Bio-Tek Co., USA). Briefly, DH5 $\alpha$ transformants of sufficiently high density that could express the target plasmid were made and lysed using the lysing solution in the kit. The plasmid DNA was isolated from the genomic DNA using the DNA-specific resin in the column and collected.

The purity of the extracted pEGFP plasmid DNA was more than adequate, given that the optical density value of $260 / 280 \mathrm{~nm}$ was 1.8 , as measured on an ultraviolet spectrophotometer (DU800, Beckman Coulter, USA). pEGFP plasmid DNA was identified using a digestive enzyme (SalI or XhoI) and subsequent electrophoresis. The map of pEGFP was analyzed and two restriction sites, SalI and XhoI, were included to verify that the obtained plasmid was pEGFP.

Detection of cell death and membrane damage. Cells were randomly assigned into four groups: a control group, without exposure to ultrasound and without the addition of SonoVue; group A, without exposure to ultrasound and with the addition of SonoVue $(200 \mu \mathrm{l} / \mathrm{ml})$; group B, exposure to ultrasound without the addition of SonoVue; and group $\mathrm{C}$, exposure to ultrasound with the addition of SonoVue $(200 \mu \mathrm{l} / \mathrm{ml})$. Each group consisted of six samples. Ultrasonically-induced cell damage was detected by using trypan blue dye $(0.4 \%)$ immediately after $5 \mathrm{~min}$ of sonication (16). Cells stained with trypan blue were counted under an optical microscope using a hemocytometer. The cell death rate was obtained from the following 


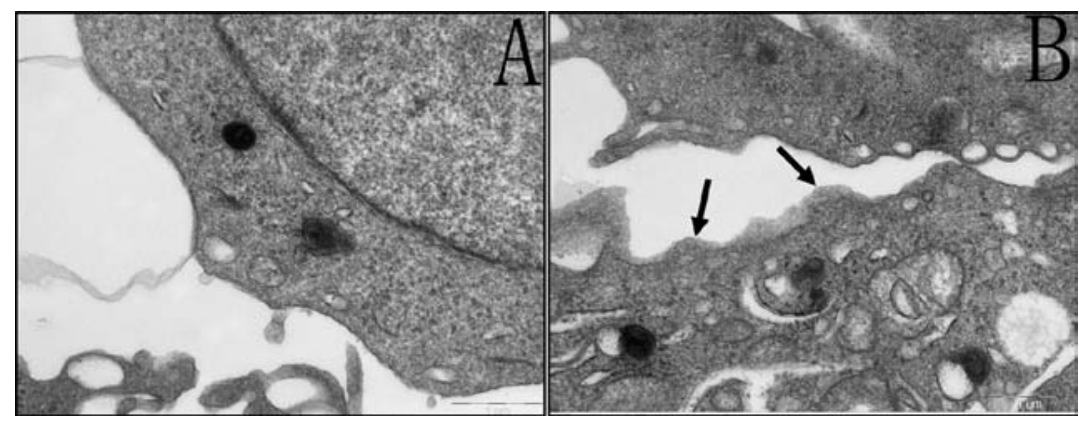

Figure 2. Representative transmission electron microscopy photomicrographs of PC-3 cell membrane damage in (A) the control group and group A and (B) in group B and C. Magnification, x24500.

equation: cell death rate $=$ the number of stained cells/the total number of cells in the group x100\%.

In order to confirm that membrane damage occurred, $50 \mu \mathrm{l}$ of fluorescein isothiocyanate (FITC)-dextran $(25 \mathrm{mg} / \mathrm{ml}$, SigmaAldrich, St. Louis, MO, USA) was added to each sample. FITC-dextran is the conjugate of fluorescein and dextran, and has a molecular weight of $70000 \mathrm{Da}$, which makes it difficult to penetrate the cellular membrane under normal conditions (17). After $5 \mathrm{~min}$ of exposure, samples were immediately washed 3 times using PBS and the fraction of fluorescencepositive cells was measured (10000 cells in each well were detected) by flow cytometry (BD FACSAria, BD Biosciences, CA, USA), with an excitation wavelength of $492 \mathrm{~nm}$ and an emission wavelength of $518 \mathrm{~nm}$ (16).

Transmission electron microscopy observation. Immediately after 5 min of sonication, the cells were observed using transmission electron microscopy (TEM).

Detection of gene transfection efficiency. To prepare the reagent of transfection according to the protocol of the Lipofectamine $^{\mathrm{TM}} 2000 \mathrm{kit}$ (Invitrogen), the ratio of plasmid DNA $(\mu \mathrm{g})$ to liposome $(\mu \mathrm{l})$ was 1:2. Before ultrasound irradiation, the reagent was added to the suspension of PC-3 cells in each sample. The PC-3 cells, at a density of $1 \times 10^{5}$ cells $/ \mathrm{ml}$, were resuspended in polystyrene sample test tubes. Diluted DNA in $100 \mu \mathrm{l}$ of DMEM without serum was mixed gently. Lipofectamine 2000 was mixed gently before use and then the appropriate amount was diluted in $100 \mu \mathrm{l}$ of DMEM without serum, followed by incubation for $5 \mathrm{~min}$ at room temperature. After $5 \mathrm{~min}$, the diluted DNA was combined with the diluted Lipofectamine 2000, mixed gently and incubated for $20 \mathrm{~min}$ at room temperature. Cells were divided into eight groups, each consisting of six samples: the control group (pEGFP), group A (SonoVue+pEGFP), group B (ultrasound+pEGFP), group C (SonoVue+ultrasound+pEGFP), group D (Lipofectamine+pEGFP+SonoVue), group E (Lipofectamine+pEGFP), group F (Lipofectamine+pEGFP+ ultrasound) and group $\mathrm{G}$ (Lipofectamine+pEGFP+ultrasound +SonoVue). In groups A, C and D, $200 \mu 1$ of SonoVue was added into each tube in each group before exposure. After 5 min of exposure, the cell suspensions were plated into 12-well plates, and after $4 \mathrm{~h}$, the serum-free medium was replaced by medium containing $10 \%$ fetal bovine serum. If the pEGFP DNA transfected into the cytoplasm, the cells would express
Table I. Cell death and membrane damage after ultrasound exposure (mean $\pm \mathrm{SD})$.

\begin{tabular}{llc}
\hline Group & $\begin{array}{c}\text { Cell death } \\
(\%)\end{array}$ & $\begin{array}{c}\text { Cell membrane } \\
\text { damage }(\%)\end{array}$ \\
\hline Control group & $0.45 \pm 0.19$ & $1.13 \pm 0.12$ \\
A (SonoVue) & $0.47 \pm 0.33$ & $1.28 \pm 0.15$ \\
B (ultrasound) & $4.98 \pm 1.23^{\mathrm{a}, \mathrm{b}}$ & $23.02 \pm 2.54^{\mathrm{a}, \mathrm{b}}$ \\
C (ultrasound+SonoVue) & $6.27 \pm 0.81^{\mathrm{a}-\mathrm{c}}$ & $41.87 \pm 2.55^{\mathrm{a}-\mathrm{c}}$
\end{tabular}

${ }^{\text {a }} \mathrm{P}<0.05$ vs. control group; ${ }^{\text {b }} \mathrm{P}<0.05$ vs. group $\mathrm{A}$ (SonoVue); ${ }^{\mathrm{c}} \mathrm{P}<0.05$ vs. group B (ultrasound).

green fluorescence and could be identified by flow cytometry. After culturing the cells for $24 \mathrm{~h}$, the pEGFP transfection efficiency was detected with fluorescence microscopy and flow cytometry.

Statistical analysis. ANOVA was used to analyze the differences in cell death and membrane damage among the four groups and the differences in gene transfection efficiency among the eight groups. $\mathrm{P}<0.05$ was considered statistically significant.

\section{Results}

Cell death and membrane damage. The results of cell death and membrane damage are presented in Table I. To assess cell death and membrane damage, the cells were examined immediately after sonication. The cell death rates induced in group B and group $\mathrm{C}$ were significantly greater than those of the control group and group A. There was no difference between the control group and group A.

Cell membrane damage was evaluated with flow cytometry. The cell membrane damage rate in group $\mathrm{C}$ was the highest of the four groups, and it was approximately 42-fold greater than the control group. The cell membrane damage rate in group B was also greater than those of the control group and group A.

Transmission electron microscopy observation. Immediately after ultrasound treatment, the cells were observed using TEM. In groups $\mathrm{B}$ and $\mathrm{C}$, there was some cell membrane damage, cell membrane discontinuity, and there were gaps in the cell 
Table II. Gene transfection efficiency of PC-3 cells after ultrasound exposure (mean \pm SD).

\section{Group}

Control (pEGFP)

A (SonoVue+pEGFP)

$\mathrm{B}$ (ultrasound+pEGFP)

$\mathrm{C}$ (SonoVue+ultrasound $+\mathrm{pEGFP})$

$\mathrm{D}$ (Lipofectamine+pEGFP+SonoVue)

$\mathrm{E}$ (Lipofectamine+pEGFP)

$\mathrm{F}$ (Lipofectamine+pEGFP+ultrasound)

G(Lipofectamine+pEGFP+ultrasound+SonoVue)
Gene transfection efficiency $(\%)$
$0.25 \pm 0.39$
$0.32 \pm 0.55$
$1.50 \pm 0.51^{\mathrm{a}, \mathrm{b}}$
$3.85 \pm 0.55^{\mathrm{a}-\mathrm{c}}$
$8.87 \pm 0.94^{\mathrm{a}-\mathrm{c}}$
$9.65 \pm 1.20^{\mathrm{a}-\mathrm{d}}$
$14.00 \pm 0.77^{\mathrm{a}-\mathrm{f}}$
$20.30 \pm 1.17^{\mathrm{a}-\mathrm{g}}$

${ }^{a} \mathrm{P}<0.05$ vs. control group; ${ }^{\mathrm{b}} \mathrm{P}<0.05$ vs. group A (SonoVue+pEGFP); ${ }^{\mathrm{c}} \mathrm{P}<0.05$ vs. group $\mathrm{B}$ (ultrasound $+\mathrm{pEGFP}$ ); ${ }^{\mathrm{d}} \mathrm{P}<0.05$ vs. group $\mathrm{C}$ (SonoVue+ultrasound+pEGFP); ${ }^{\mathrm{e}} \mathrm{P}<0.05$ vs. group D (Lipofectamine+pEGFP+SonoVue); ${ }^{\mathrm{f}} \mathrm{P}<0.05$ vs. group E (Lipofectamine+pEGFP); ${ }^{g} \mathrm{P}<0.05$ vs. group $\mathrm{F}$ (Lipofectamine+pEGFP+ultrasound).
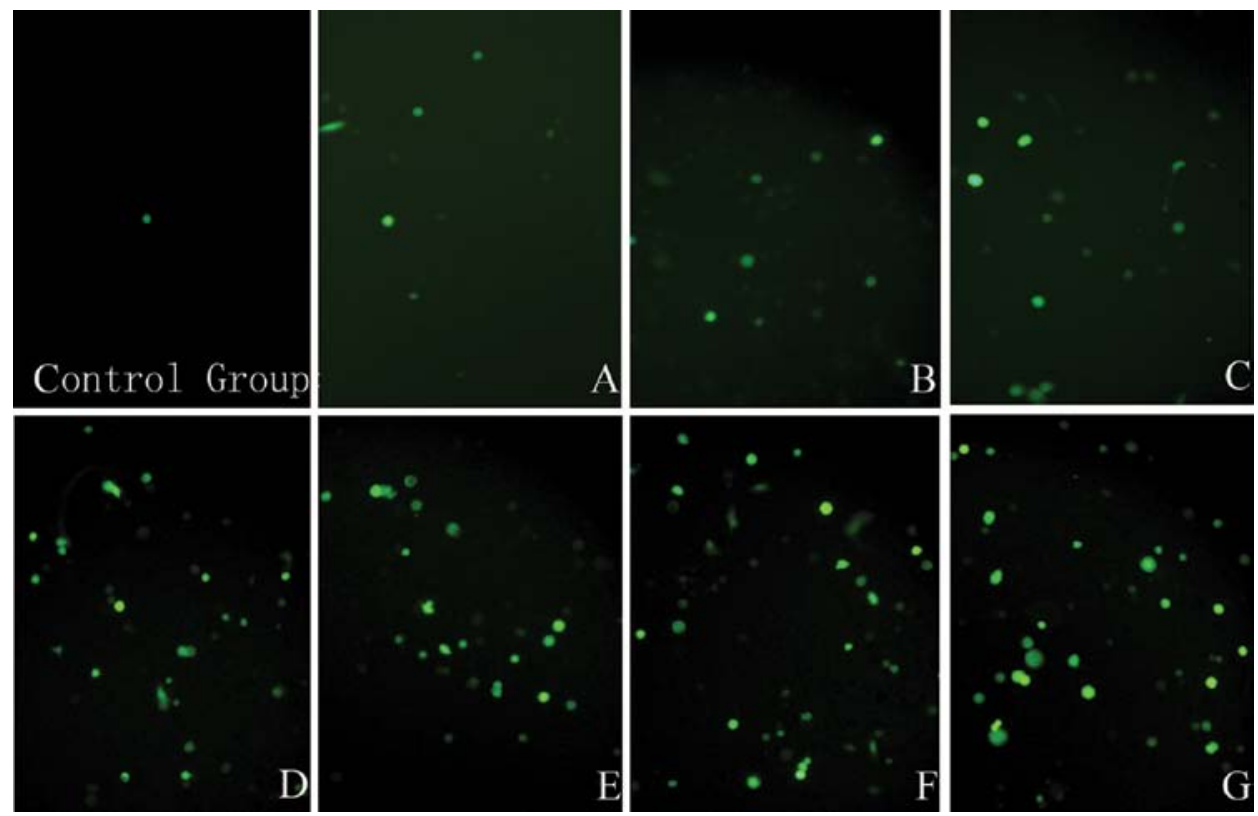

Figure 3. pEGFP expression in PC-3 cells of the control group and of groups A-G examined by fluorescence micrographs. Magnification, x20.

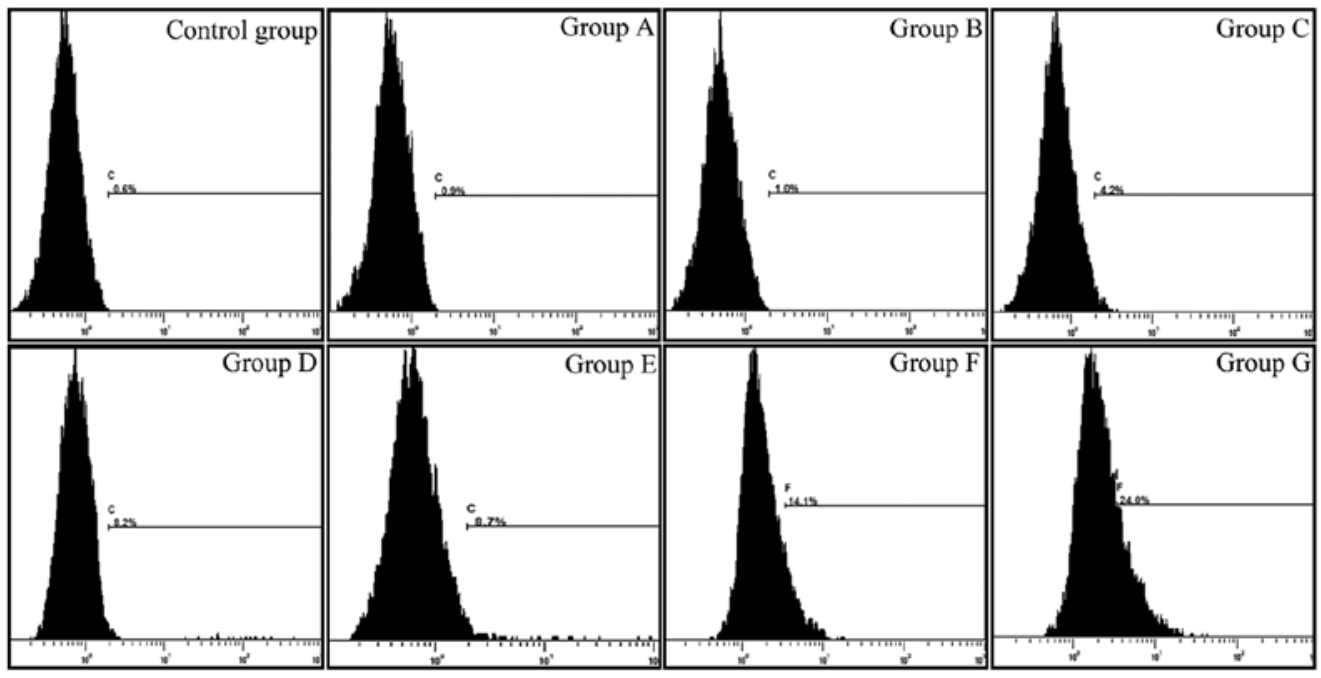

Figure 4. pEGFP expression in PC-3 cells of the control group and of groups A-G examined by flow cytometry. 
membranes (Fig. 2B). In the control group and group A, the cell membranes were continuous (Fig. 2A) and there was no cell membrane damage.

Detection of gene transfection efficiency. The results for gene transfection efficiency by flow cytometry are presented in Table II and Fig. 4. In group G (Lipofectamine+pEGFP+ultrasound+SonoVue), fluorescence microscopy revealed that the PC -3 cells transfected by the pEGFP gene had a detectable green fluorescence in the cytoplasm, while the cells in the other groups had less coloration (Fig. 3). Flow cytometry also showed that group $\mathrm{G}$ of the PC-3 cells had higher pEGFP expression than the other groups, and the transfection efficiency of group $\mathrm{G}$ was $\sim 81$-fold greater than that of the control group.

\section{Discussion}

In this study, PC-3 cells containing FITC-dextran were treated with ultrasound alone and with ultrasound and SonoVue and the results showed that ultrasound exposure alone induced permeation of FITC-dextran (23.82\%) into PC-3 cells, as detected by flow cytometry. The proportion of FITC-dextran-positive PC-3 cells increased to $41.87 \%$ after the addition of SonoVue. Using transmission electron microscopy, we also found cavitation of the cells after ultrasound exposure, with and without SonoVue. In this study, we used regular cell media that were neither degassed nor air-saturated, to avoid any changes in cells produced by these procedures. The cell media may have contained air bubbles that could produce cavitation (18). Under ultrasound exposure, microbubbles suspended in liquid can be collapsed intentionally by insonation; this collapse creates a mechanical force on the cell membrane and destroys the integrity of the adjoining cellular membrane. When microbubbles are added to the cell suspension, the cavitation effect is greater than when ultrasound is used alone. The mechanical and physical forces of ultrasound exposure will destroy cells if the energy and exposure time are beyond the tolerance limit of the culture, and these forces can even induce cell lysis, leading to death $(19,20)$. Our data indicate that ultrasound combined with SonoVue has a minimal effect on the viability of PC-3 cells. Our results imply that ultrasound combined with SonoVue is a promising microbubble-based technique for gene delivery.

In this study, we found that ultrasound combined with microbubbles improved the gene transfection efficiency of liposomes, and the efficiency was greater in group $G$ than in groups A, B, C, D, E or F or the control group. The molecular weight of pEGFP is $27000 \mathrm{Da}$, which is less than that of FITC-dextran. The amount of genetic material that entered the cells through the cavitations that were induced by ultrasound combined with microbubbles may be greater than the amount of FITC-dextran that entered under similar conditions.

In our study, we did not find that ultrasound or ultrasound combined with microbubbles had high gene transfection efficiency $(21,22)$. We believe there are various reasons for this finding. Firstly, different microbubbles have different characteristics. The nature of the shell and parcel of the gas affect the transfer capacity of the cell and different microbubbles, used with the same experimental cells and tissues, will produce different results $(23,24)$. In past studies, the microbubbles used were Levivost (25), Optison (7) and SonoVue (26). Secondly, ultrasound combined with microbubbles improved gene transfection efficiency, not only because of the induced cavitation, but also because of the influence of the microbubbles and because of DNA or RNA interactions $(27,28)$. Thus, some of our results differ from those of past studies. In the past studies, it was shown that lipid-based contrast agents are more suitable for ultrasound-mediated gene transfection than no contrast agents at all (29). Therefore, in this study, we chose SonoVue, which contains phospholipids.

In past studies, the authors usually used ultrasound frequencies between 1-3 MHz $(10,21)$. In this study, we used low-frequency and low-energy ultrasound, which has advantages such as easy penetration of the organism, less tissue absorption and less induction of tissue injury. The effects induced by low-frequency ultrasound are mainly mechanical effects and cavitation effects and the temperature increase through the thermal effect is virtually negligible (30). Given these advantages, low-frequency and low-energy ultrasound shows promise for future use in cancer therapy.

Our study showed that sonoporation, in the presence of microbubbles, is a promising technique that improved the liposome transfer of genes into prostate cancer cells, and may provide an experimental model for clinical gene therapy. Additionally, low-frequency and low-energy ultrasound that induced the destruction of microbubbles and is combined with liposomes is a feasible and efficient method of gene delivery into prostate cancer cells. Although the exact mechanisms underlying efficient gene transfection remain incompletely understood, the rapid collapse of microbubbles during sonoporation is considered to play a major role in gene delivery into cells.

\section{Acknowledgements}

This study was supported by the major infrastructure projects of Shanghai Science and Technology under grant No. 10JC1412600.

\section{References}

1. Jemal A, Siegel R, Xu J and Ward E: Cancer statistics, 2010. CA Cancer J Clin 60: 277-300, 2010.

2. Mangar SA, Huddart RA, Parker CC, Dearnaley DP, Khoo VS and Horwich A: Technological advances in radiotherapy for the treatment of localised prostate cancer. Eur J Cancer 4: 908-921, 2005.

3. Tanaka G, Hirata Y, Goldenberg SL, Bruchovsky N and Aihara K: Mathematical modelling of prostate cancer growth and its application to hormone therapy. Philos Transact A Math Phys Eng Sci 368: 5029-5044, 2010.

4. Lecornet E, Ahmed HU, Moore C and Emberton M: Focal therapy for prostate cancer: a potential strategy to address the problem of overtreatment. Arch Esp Urol 63: 845-852, 2010.

5. Baumert H: Salvage treatments for prostatic radiation failure. Cancer Radiother 14: 442-445, 2010.

6. Verma IM and Somia N: Gene therapy-promises, problems and prospects. Nature 389: 239-242, 1997.

7. Takahashi M, Kido K, Aoi A, Furukawa H, Ono M and Kodama T: Spinal gene transfer using ultrasound and microbubbles. J Control Release 117: 267-272, 2007.

8. Whelan J: Electroporation and ultrasound for gene and drug delivery. Drug Delivery Today 11: 585-586, 2002.

9. Danialou D, Corntois AS, Dudley RW, et al: Ultrasound increase plasmid-mediated gene transfer to dystrophic muscles without collateral damage. Mol Ther 5: 683-687, 2002.

10. Negishi Y, Omata D, Iijima H, et al: Enhanced laminin-derived peptide AG73-mediated liposomal gene transfer by bubble liposomes and ultrasound. Mol Pharm 7: 217-226, 2010. 
11. Audouy SA, De Leij LF, Hoekstra D and Molema G: In vivo characteristics of cationic liposomes as delivery vectors for gene therapy. Pharm Res 19: 1599-1605, 2002.

12. Hirko A, Tang F and Hughes JA: Cationic lipid vectors for plasmid DNA delivery. Curr Med Chem 10: 1185-1193, 2003.

13. Kodama T, Tomita Y,Koshiyama K and Blomley MJ: Transfection effect of microbubbles on cells in superposed ultrasound waves and behavior of cavitation bubble. Ultrasound Med Biol 32: 905-914, 2006

14. Hassan MA, Feril LB Jr, Suzuki K, Kudo N, Tachibana K and Kondo T: Evaluation and comparison of three novel microbubbles: enhancement of ultrasound-induced cell death and free radicals production. Ultrason Sonochem 16: 372-378, 2009.

15. Tabuchi Y, Takasaki I, Zhao QL, et al: Genetic networks responsive to low-intensity pulsed ultrasound in human lymphoma U937 cells. Cancer Lett 270: 286-294, 2008.

16. Hao Q, Liu Q, Wang X, Wang P, Li T and Tong WY: Membrane damage effect of therapeutic ultrasound on Ehrlich ascitic tumor cells. Cancer Biother Radiopharm 24: 41-47, 2009.

17. Ramanathan $\mathrm{M}$, Kuo HR, Lambert $\mathrm{CW}$ and Ingoglia NA: Introduction of macromolecules into synaptosomes using electroporation. J Neurosci Methods 96: 19-23, 2000.

18. Chumakova OV, Liopo AV, Evers BM and Esenaliev RO: Effect of 5-fluorouracil, Optison and ultrasound on MCF-7 cell viability. Ultrasound Med Biol 32: 751-758, 2006.

19. Kawai $\mathrm{N}$ and Iino $\mathrm{M}$ : Molecular damage to membrane proteins induced by ultrasound. Ultrasound Med Biol 29 609-614, 2003.

20. Marentis TC, Kusler B, Yaralioglu GG, Liu S, Haeggström EO and Khuri-Yakub BT: Microfluidic sonicator for real-time disruption of eukaryotic cells and bacterial spores for DNA analysis. Ultrasound Med Biol 31: 1265-1277, 2005.

21. Wang JF, Wu CJ, Zhang CM, Qiu QY and Zheng M: Ultrasoundmediated microbubble destruction facilitates gene transfection in rat C6 glioma cells. Mol Biol Rep 36: 1263-1267, 2009.
22. Manome $\mathrm{Y}$, Nakayama $\mathrm{N}$, Nakayama $\mathrm{K}$ and Furuhata $\mathrm{H}$ : Insonation facilitates plasmid DNA transfection into the central nervous system and microbubbles enhance the effect. Ultrasound Med Biol 31: 693-702, 2005.

23. Li T, Tachibana K, Kuroki M and Kuroki M: Gene transfer with echo-enhanced contrast agents: comparison between Albunex, Optison, and Levovist in mice - initial results. Radiology 229: 423-428, 2003

24. Wang X, Liang HD, Dong B, Lu QL and Blomley MJ: Gene transfer with microbubble ultrasound and plasmid DNA into skeletal muscle of mice: comparison between commercially available microbubble contrast agents. Radiology 237: 224-229, 2005.

25. Manome Y, Nakayama N, Nakayama K and Furuhata H: Insonation facilitates plasmid DNA transfection into the central nervous system and microbubbles enhance the effect. Ultrasound Med Biol 31: 693-702, 2005.

26. Nie F, Xu HX, Tang Q and Lu MD: Microbubble-enhanced ultrasound exposure improves gene transfer in vascular endothelial cells. World J Gastroenterol 12: 7508-7513, 2006.

27. Duvshani-Eshet M, Adam D and Machluf M: The effects of albumin-coated microbubbles in DNA delivery mediated by therapeutic ultrasound. J Control Release 112: 156-166, 2006.

28. Duvshani-Eshet M, Baruch L, Kesselman E, Shimoni E and Machluf M: Therapeutic ultrasound-mediated DNA to cell and nucleus: bioeffects revealed by confocal and atomic force microscopy. Gene Ther 13: 163-172, 2006.

29. Watanabe A, Otake R, Nozaki T, et al: Effects of microbubbles on ultrasound-mediated gene transfer in human prostate cancer PC3 cells: comparison among Levovist, YM454, and MRX-815H. Cancer Lett 265: 107-112, 2008.

30. Samuel S, Miller DL and Fowlkes JB: The relationship of acoustic emission and pulse-repetition frequency in the detection of gas body stability and cell death. Ultrasound Med Biol 32: 439-447, 2006 